\title{
La siesta y el exceso de peso en escolares de Mérida, Yucatán. Estudio analítico transversal
}

Méndez-Domínguez $\mathrm{N}^{1}$, Prelip $\mathrm{M}^{2}$, Aguilar-Vargas $\mathrm{E}^{3}$, Avilés-lbarra $\mathrm{O}^{3}$, Dic-
kinson $\mathrm{F}^{4}$

Resumen

INTRODUCCIÓN: en Mérida, Yucatán, México, la siesta es una costumbre arraigada que hace referencia al período dedicado a dormir después de almorzar.

OBJETIVO: describir el hábito de la siesta y su asociación con el exceso de peso en los escolares de primaria.

MATERIALES Y MÉTODOS: estudio transversal prolectivo desarrollado en 2012 en una muestra probabilística de 2,104 escolares procedentes de 16 escuelas públicas aleatoriamente seleccionadas en Mérida, Yucatán.

RESULTADOS: se observó que los escolares que hacen siesta $(\geq 3$ veces/semana, $\geq 2$ horas/día) son más propensos al exceso de peso $(z=2.73, p=0.01)$; realizan significativamente menos ejercicio $(z=-5.91, p<0.00)$; duermen menos durante la noche $(z=-17.59$, $p=0.01)$ y consumen menos frutas y verduras $(z=-2.59, p=0.01)$. También ven más horas de televisión.

DISCUSIÓN: la costumbre de la siesta, a pesar de ser un hábito muy arraigado, debe ser limitada en tiempo, para no afectar el estado nutricional de los escolares meridanos al favorecer el sedentarismo.

PALABRAS CLAVE: sueño, sedentarismo, sobrepeso, índice de masa corporal.

Acta Pediatr Mex. 2017 May;38(3):143-151.

\section{Nap and overweight in schoolchildren from Merida, Yucatan: A cross-sectional analytical study.}

Méndez-Domínguez $\mathrm{N}^{1}$, Prelip $\mathrm{M}^{2}$, Aguilar-Vargas $\mathrm{E}^{3}$, Avilés-Ibarra $\mathrm{O}^{3}$, Dickinson $\mathrm{F}^{4}$

\section{Abstract}

INTRODUCTION: In Merida, Yucatan, Mexico, the afternoon nap is a deep-rooted habit that makes reference to a period dedicated to sleep after lunch.
${ }^{1}$ Doctora en Ciencias de la Salud, Maestra en Ecología Humana, Médico Cirujano. Investigadora Posdoctorante en el Centro de Investigación y de Estudios Avanzados del IPN. Unidad Mérida. Departamento de Ecología Humana. Profesora titular en la escuela de Medicina de la Universidad marista de Mérida. ${ }^{2}$ Doctor en Administración Pública, Maestro en Salud Pública. Escuela de Salud Pública Fielding, Universidad de California Los Ángeles, Estados Unidos. Decano Asociado, Profesor en residencia, Director de estudios de campo en el Departamento de Ciencias de la Salud Comunitaria.

${ }^{3}$ Médico Interno de Pregrado. Escuela de Medicina. Universidad Marista de Mérida.

${ }^{4}$ Doctor en Ciencias Naturales. Maestro en Antropología. Investigador Titular. Centro de Investigación y de Estudios Avanzados del IPN. Unidad Mérida. Departamento de Ecología Humana.

Recibido: 12 de julio del 2016

Aceptado: 29 de septiembre del 2016

\section{Correspondencia}

Nina Méndez-Domínguez

ninuxka@hotmail.com

Este artículo debe citarse como

Méndez-Domínguez N, Prelip M, Aguilar-Vargas E, AvilésIbarra O, Dickinson F. La siesta y el exceso de peso en escolares de Mérida, Yucatán. Estudio analítico transversal. Acta Pediatr Mex. 2017;38(3):143-151. 
OBJECTIVE: Of this observational study is to describe the habit of the afternoon nap and its association with overweight among schoolaged children.

METHODS: This is a section cross sectional prospective study done in 2012 in a probability sample of 2,104 school children of 16 public schools randomly selected in Merida, Yucatan.

RESULTS: Children who were accustomed to take after lunch naps $(\geq 3 / \geq 2$ days/hours during week days) were more prone to be overweight $(z=2.73, p=0.01)$ than their peers; they also performed significantly less physical exercise $(z=-5.91, p<0.00)$; slept less at night $(z=-17.59, p=0.01)$ and ateless fruits and vegetables $(z=-2.59$, $p=0.01)$; as they watch more hours of television.

DISCUSSION: The after lunch nap, despite being a deeply rooted habit, should not last too long, in order to reduce the sedentary time and to promote healthy habits among school aged children.

KEYWORDS: sleep; sedentary behavior; overweight; body mass index
Correspondence Nina Méndez-Domínguez ninuxka@hotmail.com

\section{INTRODUCCIÓN}

Al período dedicado a dormir después del almuerzo se le conoce como siesta. Ésta no es un evento ocasional, sino una costumbre arraigada culturalmente que se mantiene a través de las generaciones y forma parte de la rutina diaria de muchas familias en el mundo, particularmente en las regiones tropicales y calurosas situadas entre los $30^{\circ}$ al norte y $30^{\circ}$ al sur del ecuador. ${ }^{1,2}$

La siesta, vista por parte de los integrantes de las culturas que no la practican, suele percibirse como un signo de flojera o falta de ambición, característico de las Ilamadas "culturas de la siesta" ${ }^{1,3}$

Yucatán es un estado situado al sureste de México, bañado por las aguas del Golfo de México. Mérida, su capital, es una de las ciudades regionales que en las últimas cuatro o cinco décadas ha tenido mayor crecimiento urbano y demográfico, asociado a la inmigración, tanto desde las zonas rurales del estado, como de otras áreas de México y otros países, y que ha dado lugar a cambios diversos en el ambiente sociocultural de Mérida en el que coexisten los hábitos arraigados en la sociedad yucateca tradicional y las tendencias globalizadoras que comúnmente dificultan la permanencia de las tradiciones, entre ellas, el hábito de la siesta. ${ }^{4}$

Desde antes del crecimiento acelerado de la capital yucateca, la siesta era un tiempo incorporado a la vida de las "haciendas" del siglo XVII, varias de ellas localizadas en la que hoy es la ciudad de Mérida, a tal grado que el realizar visitas o planear actividades durante la preciada hora de la siesta era considerado como una falta a la buena educación. ${ }^{5}$

En esa época, la siesta también se practicaba en la ciudad de Mérida, como se menciona en el siguiente fragmento: “Después del bochorno del 
medio día -cuando toda la familia se levantaba de dormir la siesta y si no se tenía pendiente realizar alguna visita- la familia en pleno se volcaba al exterior". ${ }^{6}$

De este fragmento retomamos dos puntos importantes:

1. La siesta se realizaba en las primeras horas de la tarde cuando la temperatura es tan alta que prácticamente imposibilita la realización de actividades en el exterior.

2. La hora de la siesta era seguida de un periodo activo de recreación en el exterior de la vivienda, tanto para los niños como para los adultos. ${ }^{6}$

Los cambios derivados de la globalización en la Mérida contemporánea presentan nuevos retos para la permanencia de la costumbre de la siesta, derivados de la adopción de estilos de vida de las ciudades más industrializadas, en donde no hace tanto calor entre las 13:00 y 15:00 horas o donde los sitios de trabajo y hogares cuentan con sistemas de aire acondicionado. Entre las razones por las cuales se adoptan estos horarios se encuentra la consideración de que dejar tiempo para la siesta afectaría la productividad de los trabajadores. ${ }^{7,8}$

En muchos centros de educación preescolar de Yucatán se ha implementado la extensión del tiempo de estancia de los niños, los cuales pueden permanecer en las instalaciones hasta cuatro o cinco de la tarde (en el horario tradicional de educación la jornada dura de 8 a 11 horas). Sin embargo, el nuevo horario, cuando implica la suspensión de las siestas de los niños, no es bien aceptado por los padres de familia quienes han insistido en que se respete el tiempo destinado a la siesta aún durante su estancia en la escuela. Este es un ejemplo de cómo incluso parejas jóvenes con hijos pequeños mantienen el arraigo por la siesta y fomentan su práctica, por lo que, para la edad escolar, los niños la han hecho parte de su vida diaria. ${ }^{9}$

La siesta en Mérida es practicada no sólo por la población local, sino también por personas que migran temporalmente a la ciudad e incluso por turistas que la visitan por periodos cortos. ${ }^{10}$

El término inglés napen hace referencia a un período breve en el cual se duerme, que suele durar de 30 a 45 minutos, pero a diferencia del napen, la siesta suele durar más. En el caso de los niños de primaria, que suelen salir de los colegios entre las 11:30 y 12:30 horas y que almuerzan alrededor de las 12:00 o 13:00 horas, suelen tomar siesta hasta las 16. Esta duración, de tres horas (pero no menor a dos) se ha descrito tanto en Mérida como en otras comunidades de Yucatán. ${ }^{11,12}$

\section{OBJETIVO}

Describir inicialmente el hábito de la siesta en el ámbito de la capital yucateca y cómo se lleva a cabo por los escolares de primaria para, finalmente, analizarlo en función de la epidemia de sobrepeso y obesidad que aqueja a la sociedad yucateca, mucho más que a otras poblaciones de México.

\section{MATERIAL Y MÉTODOS}

Este proyecto fue aprobado por el comité de ética de la Facultad de Medicina de la Universidad Autónoma de Yucatán. Todos los participantes otorgaron su consentimiento informado bajo firma con testigo y en el caso de los escolares, el padre, madre o tutor legal proporcionó el consentimiento; adicionalmente, todos los escolares incluidos en el presente estudio otorgaron su asentimiento verbal. 


\section{Entrevista}

Este artículo deriva de un estudio de metodología mixta, con diseño exploratorio, en el cual los aspectos obtenidos mediante métodos cualitativos fueron las bases para un estudio cuantitativo. ${ }^{13}$

Durante la fase inicial cualitativa exploratoria, el primer paso fue generar una guía de entrevista para grupos focales, que inicialmente se validó con doce madres de familia en una comunidad comparable a la comunidad blanco. Posteriormente, se desarrollaron los grupos focales con participación de padres de familia y cuidadores primarios de niños en edad escolar. ${ }^{14}$

Tras la validación del cuestionario se generaron seis grupos focales con participación de 48 cuidadoras principales de escolares con obesidad morbosa y, en base a las hipótesis obtenidas a partir del análisis de su discurso, se formuló una guía de entrevista que fue validada y posteriormente aplicada a los padres de familia de los niños enrolados en las escuelas o, en su defecto, durante la entrega de los resultados antropométricos de los escolares a sus padres o cuidadores principales. Respecto a los hábitos de sueño, para el presente artículo se toma en cuenta como variable dependiente al hábito de dormir siesta $\geq 2$ horas diarias por tres o más días entre semana (lunes a viernes).

\section{Antropometría}

Este fue un estudio transversal realizado durante el año escolar 2012-2013. El tamaño de la muestra, calculada para ser representativa de los 70,780 alumnos entre los seis y los doce años de edad que acudían a las primarias públicas de la ciudad de Mérida, Yucatán, fue de 2,104 individuos (1,121 niñas y 983 niños). En base a este universo, se estimó una muestra de 2,104 estudiantes, tomando en cuenta la última prevalencia conocida de sobrepeso/obesidad, un $95 \%$ de confianza y $5 \%$ de error máximo y conservando la razón mujer/hombre 1.13/1 conocida para la ciudad de Mérida 38\%. Para la selección aleatoria de las 16 escuelas en que se realizó el estudio, se empleó la lista oficial de escuelas primarias públicas situadas dentro de la carretera periférica que marca los límites de la ciudad. ${ }^{15-17}$

El equipo de trabajo que realizó las antropometrías fue capacitado previamente y llevó a cabo un proceso de estandarización, para evitar las variaciones entre observadores. Las mediciones se realizaron en las escuelas en salones aclimatados específicamente para este propósito. Las mediciones fueron realizadas a la misma hora del día, para evitar sesgos. Se utilizó una báscula digital, con 100 gramos de precisión (marca Seca ${ }^{\circledR}$, modelo 876 ), para pesar a los participantes quienes llevaban la menor ropa posible y sin zapatos (proporcionamos ropa ligera cuando fue necesario). La estatura se midió con un estadiómetro portátil (Seca ${ }^{\circledR}$, modelo 206) con precisión de 1 milímetro siguiendo los procedimientos estándar descritos por Lohman y sus colaboradores, con los participantes en posición erguida, respetando el plano horizontal de Frankfurt. ${ }^{18}$

Con base en los hallazgos de la fase cualitativa, descrita en la sección previa, se identificó que el hábito de dormir siesta se define culturalmente a dormir dos o más horas después de la hora del almuerzo, tres o más veces por semana, lo que permitió clasificar a quienes cumplen con estos criterios como que poseen el hábito de la siesta (variable dependiente).

Para efectos del presente estudio, se consideraron los percentiles del índice de masa corporal, que estandarizan para edad y sexo la puntuación en la cual se encuentran los menores de edad en una escala del cero al cien. De acuerdo a los parámetros de la Organización Mundial de la 
Salud un percentil entre 85-94.9 corresponde a sobrepeso y una igual o mayor a 95 corresponde a obesidad; en este estudio empleamos la variable independiente "exceso de peso" para referirnos a cualquier percentil igual o superior al 85, la cual fue analizada de manera dicotómica, (presencia o ausencia de exceso de peso). ${ }^{19}$

Entre las variables de ajuste y co-variables se incluyen:

Sexo (Masculino/Femenino); grado escolar (de primero a sexto); horas de ejercicio por semana (variable numérica, continua); horas efectivas de sueño nocturno (numérica continua); horas de tele entre semana (numérica, continua); consumo de refrescos gasificados en el almuerzo (dicotómica: presencia/ausencia) y días de consumo de frutas y verduras a la semana (numérica, discreta).

\section{Análisis de datos}

La información fue vaciada y codificada en una hoja de cálculo y procesada posteriormente empleando el programa Stata 12. Se obtuvo la estadística descriptiva de la muestra, se realizaron comparaciones de medias empleando la prueba t de Student por sexo y se corrió un análisis de regresión logística multinomial. Se consideraron significativos parámetros con valor de $\mathrm{p}<0.05$.

\section{RESULTADOS}

Los 2,104 alumnos fueron medidos y pesados para obtener su información antropométrica. Un solo escolar por hogar fue incluido en este estudio.

El promedio de horas de siesta para la muestra completa fue de $1.69 \pm 0.03$ (IC 95\%: 1.63-1.75) para las mujeres, y de $1.78 \pm 0.03$ (IC 95: 1.631.75) para los varones $(t=1.76 ; p=0.039) ; 1,414$ escolares (67.2\%) tienen el hábito de dormir al menos dos horas diarias, tres veces por semana. Ni el tiempo de siesta, ni la proporción de participantes que duermen siesta mostraron diferencias significativas por edad o grado escolar $(p>0.05)$.

El promedio de percentil de índice de masa corporal en la muestra, fue de $76.6 \pm 0.61$ (IC 95\%: 75.4-77.76) y la proporción de individuos con exceso de peso fue de $50.05 \%$. Se puede observar la diferencia en proporción de participantes con exceso de peso por sexo en el Cuadro 1.

El percentil de índice de masa corporal fue significativamente mayor en quienes tienen el hábito de dormir siesta (77.4 \pm .73 , IC 95\%: 76.0-78.9), cuando se compararon con aquellos que no lo tienen $(74.7 \pm 1.1$, IC 95\%: 29.0 72.5-76.9). En el Cuadro 2, se presenta el modelo de análisis de regresión logística (ajustado por sexo) que muestra la asociación entre los hábitos de la siesta con las variables analizadas.

El modelo de regresión muestra que existe una asociación directa y significativa entre el exceso de peso y tomar siesta de dos o más horas, al menos tres veces por semana. Así mismo, los escolares que poseen el hábito de la siesta, realizan menos ejercicio, duermen menos durante la noche y consumen con menor frecuencia frutas y verduras. Por otro lado, quienes toman siesta larga, pasan más horas semanales viendo televisión.

\section{DISCUSIÓN}

Hemos descrito los patrones de siesta e identificado su asociación significativa con el exceso de peso y los hábitos saludables de estudiantes que asisten a escuelas primarias públicas de la ciudad de Mérida. Previamente, con una muestra de escolares de secundaria de Taiwan, Chen y sus colaboradores identificaron la asociación entre la reducción del sueño nocturno con hábitos poco saludables, ya que quienes duermen más 
Cuadro 1. Comparación de las características de los participantes por sexo

\begin{tabular}{|l|c|c|c|c|c|c|c|}
\hline Con hábito de dormir siesta & Mujeres & DE & IC 95\% & Hombres & DE & IC 95\% & Valor p \\
\hline Horas de ejercicio por semana & 1.78 & 2.46 & $1.62-1.93$ & 1.88 & 2.48 & $1.73-2.02$ & 0.171 \\
\hline Horas de sueño nocturno & 8.22 & 1.09 & $8.15-8.29$ & 8.22 & 1.07 & $8.15-8.28$ & 0.542 \\
\hline Horas de tele a la semana & 15.04 & 6.35 & $14.65-15.44$ & 14.75 & 0.19 & $6.40-14.38$ & 0.151 \\
\hline Exceso de peso* & 0.54 & 0.01 & $0.51-0.56$ & 0.47 & 0.01 & $0.44-0.49$ & $\mathbf{0 . 0 0 3}$ \\
\hline Refresco gasificado en almuerzo* & 0.63 & 0.01 & $0.60-0.66$ & 0.63 & 0.02 & $0.60-0.66$ & 0.798 \\
\hline Frutas y verduras (días/semana) & 4.97 & 1.84 & $4.68-5.08$ & 4.98 & 1.83 & $4.87-5.10$ & 0.433
\end{tabular}

DE: desviación estándar; IC: intervalo de confianza; * Indica la proporción.

Cuadro 2. Regresión logística expresando la fuerza de asociación entre la siesta y las variables presentadas $(n=2,104)$

\begin{tabular}{|c|c|c|c|c|c|}
\hline Siesta & $\mathbf{R M}$ & DE & $\mathbf{Z}$ & IC $95 \%$ & $\begin{array}{c}\text { Valor } \\
p\end{array}$ \\
\hline $\begin{array}{l}\text { Sexo } \\
\text { (masculino) }\end{array}$ & 0.99 & 0.11 & -0.11 & $0.80-1.22$ & 0.91 \\
\hline Exceso de peso & 1.35 & 0.15 & 2.73 & $1.08-1.67$ & 0.01 \\
\hline $\begin{array}{l}\text { Horas de ejerci- } \\
\text { cio por semana }\end{array}$ & 0.88 & 0.02 & -5.91 & $0.84-0.92$ & 0.00 \\
\hline $\begin{array}{l}\text { Horas de } \\
\text { sueño nocturno }\end{array}$ & 0.36 & 0.02 & -17.59 & $0.32-0.41$ & 0.00 \\
\hline $\begin{array}{l}\text { Horas diarias } \\
\text { de televisión }\end{array}$ & 1.05 & 0.01 & 5.74 & $1.03-1.07$ & 0.00 \\
\hline $\begin{array}{l}\text { Refresco } \\
\text { gasificado en } \\
\text { almuerzo }\end{array}$ & 0.91 & 0.11 & -0.80 & $0.71-1.15$ & 0.42 \\
\hline $\begin{array}{l}\text { Frecuencia } \\
\text { de frutas y } \\
\text { verduras }\end{array}$ & 0.93 & 0.03 & -2.59 & $0.87-0.98$ & 0.01 \\
\hline
\end{tabular}

RM: razón de momios; DE: desviación estándar; IC: intervalo de confianza.

horas durante la noche, llevan una mejor dieta y practican más ejercicio. ${ }^{20}$

En el presente estudio, observamos que tener el hábito de la siesta ( $\geq 3$ días a la semana por $\geq 2$ horas) se asocia en los escolares meridanos a un menor tiempo de sueño nocturno. SayonOrea y sus colaboradores, en su estudio de la cohorte SUN enuncian dos vías por las cuales el dormir menos por la noche se asocia a exceso de peso: 1) dormir menos de lo necesario tiene un impacto en las hormonas implicadas en la regulación del apetito; lo que se traduce en niveles más bajos de leptina (hormona inhibidora de apetito) y mayores niveles de grelina (hormona estimulante de apetito); 2) Que menos tiempo de dormir, trae como consecuencia mayor tiempo para comer, pues aún se está despierto. ${ }^{21}$ Sin embargo, en el caso de los escolares de nuestra muestra, ese tiempo que no duermen por la noche, es posiblemente el que duermen en el día, con la diferencia de que, durante el día, el aprovechamiento de las hormonas que favorecen el metabolismo y el crecimiento longitudinal no es equivalente al de la noche, lo cual favorece el metabolismo bajo y el aumento de peso. ${ }^{22,23}$

Existen estudios que muestran que en los escolares la duración del sueño nocturno es menor en presencia de hábitos como ver la televisión por la noche o por el tipo de dieta habitual, ${ }^{24-26}$ mismos que a su vez, estuvieron claramente asociados a los hábitos de siesta.

Al ver televisión por muchas horas se comprometen otras actividades de mayor gasto energético, como serían los juegos y cuanto más tiempo invierten los individuos en algún tipo de ocio sedentario, es menor el que dedicarán a la actividad física. ${ }^{27,28} \mathrm{El}$ ocio sedentario predice, de un modo directo, un índice de masa corporal elevado ${ }^{29}$ Aún con la tendencia natural al movimiento, los niños actualmente poco a poco se 
están volviendo menos activos; de hecho, en un estudio que compara a los niños actuales con los de hace 50 años revela que en la actualidad, los niños gastan casi $600 \mathrm{kcal}$ diarias menos como media. ${ }^{30}$ Además, en conjunto, la publicidad presentada en la TV y dirigida a los niños se centra en productos industrializados carentes de un valor nutricio adecuado y ofrece poca o nula promoción al consumo de frutas y verduras dando lugar a que los niños reclamen con mayor frecuencia productos que son anunciados $y$, por tanto, a que aumente la ingesta de botanas y otros alimentos, más altos en energía, azúcar, sodio, grasa total y grasa saturada, lo cual se refleja en el aumento del consumo energético. ${ }^{31}$

Si vemos los hábitos de los participantes en nuestro estudio como una costumbre que resta parte del día a desarrollar actividades como hacer ejercicio, entenderemos que la siesta es no sólo una costumbre sedentaria que inhibe que los escolares tengan desgaste (derivado del ejercicio) que es el que genera cansancio y sueño por la noche. La falta de desgaste acarrea que aún a altas horas de la noche, cuando el pasatiempo más común es ver televisión, los niños se encuentren despiertos. ${ }^{32,33}$

Por otro lado, un estudio de la asociación de horas de sueño, siesta y factor de riesgo de obesidad, estableció que los sujetos que dormían menos de siete horas por noche y tomaban una siesta mayor a 30 minutos presentaban un mayor riesgo de obesidad, en comparación a sujetos que dormían de siete a ocho horas con una siesta menor a 30 minutos; además, que tomar una siesta menor a 30 minutos fue un factor protector contra la obesidad en comparación al grupo que no realizaba siesta. ${ }^{21}$ Ello nos indica que algo que pudiera ser provechoso para la salud (la siesta) al favorecer un peso saludable, cuando es demasiado larga tiene un efecto contrario, pues afecta la actividad física y reduce el tiempo de sueño nocturno.
La obesidad en los países desarrollados y en desarrollo, que se ha incrementado alarmantemente durante los últimos años, representa el principal problema de malnutrición en adultos y se ha intensificado notoriamente en la población infantil. La edad escolar y la adolescencia son unas etapas críticas para la conformación de estilos de vida y hábitos alimenticios beneficiosos que persistirán en etapas posteriores, es por ello que en esta etapa deben fortalecerse los hábitos saludables. ${ }^{34,35}$

Las complicaciones de salud provenientes de la obesidad son amplias, tanto en la edad adulta como en la infancia. Por otro lado, la obesidad infantil es la antesala de la obesidad adulta, pues se ha señalado que casi ocho de cada diez niños rollizos terminan convirtiéndose en adultos obesos. ${ }^{29}$ Por ello, es importante abordar correctamente desde etapas muy tempranas la promoción de hábitos, incluyendo los del sueño diurno y nocturno.

\section{CONCLUSIÓN}

De ningún modo es deseable afectar una costumbre tan arraigada como la siesta; sin embargo, es importante generar medidas que permitan informar a la sociedad que es recomendable que la siesta dure menos de una hora o, en su defecto, menos de dos horas y que sea seguida por actividad física, por el juego activo o la práctica de un deporte, más nunca por pasatiempos que impliquen permanecer acostado, pues ello podría afectar la hora en la que los escolares se acuesten y reducen sus oportunidades de actividad física.

\section{LIMITACIONES}

El estudio solamente es representativo de la población meridana entre 6-12 años de edad que acude a las primarias públicas. La duración de la siesta podría variar según la época del año. Al ser un estudio de corte transversal no refleja 
una relación causal entre la siesta y el exceso de peso, únicamente su asociación.

\section{AGRADECIMIENTOS}

A Ana Andrade por su apoyo técnico y logístico.

\section{REFERENCIAS}

1. Campos H, Siles X. Siesta and the risk of coronary heart disease: results from a population-based, case-control study in Costa Rica. International Journal of Epidemiology. 2000;29(3):429-37. doi: 10.1093/ije/29.3.429.

2. Valencia-Flores M, Castano VA, Campos RM, Rosenthal L, Resendiz $\mathrm{M}$, Vergara $\mathrm{P}$, et al. The siesta culture concept is not supported by the sleep habits of urban Mexican students.J Sleep Res. 1998;7(1):21-9.

3. De Grazia S. Tres conceptos antiguos en el mundo moderno: el Trabajo, el Tiempo, el Ocio. Revista de Estudios Políticos. 1963;(131):5-20.

4. Sweitz SR. A Theoretical Context for Documenting Social Change in Yucatán, Part 2: Culture Change, Social Stratification, and Power Relationships. On the Periphery of the Periphery: Springer; 2012. p. 35-52.

5. Patch RW. Maya Revolt and Revolution in the Eighteenth Century: Armonk, NY, EEUU; 2003;170pp.

6. Arana López, GN. Espacios, sujetos y objetos del habitar cotidiano en el México de entre siglos. Mérida la de Yucatán, 1886-1916. Memoria y Sociedad. 2013;17(35):236-261.

7. Coester A. The "Danza de los conquistadores" at Chichicastenango. Hispania. 1941;24(1):95-100.

8. Colley K. Coming to know a school culture. Tesis en opción al grado de Doctora en Educación. Virginia, E.E.U.U.1999; $163 \mathrm{pp}$.

9. De la Garza L, Zermeño M. Caso de estudio: Jardín de Niños Zazil Kuxtal. Revista de Investigación Educativa de la Escuela de Graduados en Educación. 2014;4(8):73-9.

10. Johanson $L$. The implementation of a study abroad course for nursing. Nurse Educ. 2006;31(3):129-31.

11. Koesler R,Watters R. Proceedings of the 1995 International Conference on Outdoor Recreation and Education (Ithaca, New York, October 26-28, 1995): ERIC; 1996.

12. Ashton GT. 17 Town-city circulation among young Yucatec shoemakers. Circulation in Third World Countries. 1985:350 pp.

13. Creswell JW, Fetters MD, Ivankova, N. Designing a mixed methods study in primary care. Ann Fam Med. 2004;2(1):712.

14. Mendez N, Barrera-Perez MA, Palma-Solis M, Dickinson F, Uicab-Pool G, Castillo Burguete MT, et al. "...You are not fat, you are hermosa": Mexican caregivers share their perceptions about their role supporting their morbidly obese children. Hisp Health Care Int. 2014;12(4):174-182.

15. Instituto Nacional de Salud Pública. Encuesta Nacional de Salud y Nutrición 2012. Resultados por entidad federativa, Yucatán, 2013. Disponible en: encuestas.insp.mx. Retrieved from Cuernavaca, México:

16. INEGI. Información Geográfica del Estado de Yucatán: Instituto Nacional de Geografía e Informática; 2010 [Actualizado en diciembre 2013, citado el 27 de junio de 2016].

17. Sistema Nacional de Información Educativa http://www. snie.sep.gob.mx/estadisticas_educativas.html: SEP; [Actualizado en enero 2016, citado el 27 de junio de 2016].

18. Lohman TG, Roche AF, Martorell R. Anthropometric standardization reference manual. Chicago, Illinois: Human Kinetics Books Champaign, III; 1991;90pp

19. De Onis M, Onyango AW, Borghi E, Siyam A, Nishida C, Siekmann J. Development of a WHO growth reference for school-aged children and adolescents. Bull World Health Organ. 2007;85(9):660-7.

20. Chen MY, Wang EK, Jeng YJ. Adequate sleep among adolescents is positively associated with health status and health-related behaviors. BMC PublicHealth. 2006;6(1):1.

21. Sayón-Orea C, Bes-Rastrollo M, Carlos S, Beunza JJ, Basterra-Gortari FJ, \& Martínez-González, MA (2013). Association between sleeping hours and siesta and the risk of obesity: The SUN Mediterranean Cohort. Obes Facts. 2013;6(4):337-347.

22. Kim TW, Jeong J-H, Hong S-C. The Impact of Sleep and Circadian Disturbance on Hormones and Metabolism. International Journal of Endocrinology. 2015;9. doi: 10.1155/2015/591729.

23. Knutson, KL (2012). Does inadequate sleep play a role in vulnerability to obesity? American Journal of Human Biology. 2012;24(3):361-371.

24. Owens J, Maxim R, McGuinn M, Nobile C, Msall M, Alario A. Television-viewing habits and sleep disturbance in school children. Pediatrics. 1999; 104(3):e27.

25. Borghese MM, Tremblay MS, Katzmarzyk PT, Tudor-Locke C, SchunaJ. M., Leduc G, Chaput JP. Mediating role of television time, diet patterns, physical activity and sleep duration in the association between television in the bedroom and adiposity in 10 year-old children. Int J BehavNutr Phys Act.2015; 12(1):2-10.

26. Peuhkuri K, Sihvola N, Korpela R. Diet promotes sleep duration and quality. NutritionResearch. 2012;32(5):309-19.

27. T Durá-Travé, \& Sánchez-Valverde, V. Obesidad infantil: ¿Un problema de educación individual, familiar o social? Acta Pediatr Esp. 2005;63(3):204-207.

28. Vázquez IA, Zapico RB, Díez JH, Rodríguez CF. Actividad física, ocio sedentario, falta de sueño y sobrepeso infantil. Psicothema. 2008;20(4):516-20.

29. Loaiza S, Atalah E. Factores de riesgo de obesidad en escolares de primer año básico de Punta Arenas. RevChilPediatr. 2006;77(1):20-6. 
30. Ara I, Vicente-Rodríguez G, Moreno LA, Gutin B, Casajus J. La obesidad infantil se puede reducir mejor mediante actividad física vigorosa que mediante restricción calórica. ApuntsMedEsport. 2009;44(163):111-8.

31. Pérez-Salgado D, Rivera-Márquez JA, Ortiz-Hernández L. (2015) Publicidad de alimentos en la programación de la televisión mexicana: ¿los niños están más expuestos? SaludPublica Mex. 2010;52(2):119-26.

32. Nuutinen T, Ray C, Roos E. Do computer use, TV viewing, and the presence of the media in the bedroom predict school-aged children's sleep habits in a longitudinal study? BMC Public Health.2013;13(1):2-8
33. Carson V, Tremblay MS, Chaput JP, Chastin SF. Associations between sleep duration, sedentary time, physical activity, and health indicators among Canadian children and youth using compositional analyses 1. ApplPhysiolNutrMetab. 2016;41(6):S294-S302.

34. Karnik S, KanekarA. Childhood obesity: a global public health crisis. Int J Prev Med. 2012;3(1):1-7.

35. Persch AC, Lamb AJ, Metzler CA, Fristad MA. Healthy habits for children: Leveraging existing evidence to demonstrate value. Am J Occup Ther. 2015; 69(4):1-5. 